\title{
Design Method of SvcV5 in DoDAF2.0 Based on Service Discovery
}

\author{
Xiaoxue Zhang ${ }^{1+}$, Gang $\mathrm{Liu}^{2}$, Aimin Luo ${ }^{1}$ and Junxian Liu ${ }^{1}$ \\ ${ }^{1}$ Science and Technology on Information Systems Engineering Laboratory, National University of Defense \\ Technology, Changsha, China \\ ${ }^{2}$ ChongQing Communication Institute, Chongqing, China
}

\begin{abstract}
During architecture design process, designers need to use specific methods to design architecture data and products. As the standards of architecture framework, DODAF2.0 lack of specific methods to design data and products. As the link of operational viewpoint and service viewpoint, SvcV5 is the key to design the service view data and products. In this paper, we studied the assistant design method of SvcV5 based on service discovery. Firstly, the relevant data is extracted from OV6b, CV4 and CV6, and the dependency matrix of activities is established based on the analysis of capability dependency and data dependency of activities. Secondly, based on the information exchange relationship of activity, Service initial discovery method is proposed; thirdly, the service cohesion and the coupling degree related metrics are analyzed, and an algorithm is used to cluster and improve the service composition. Finally, based on the mapping between result of service clustering and the list of common services, the service determination is realized to support the design of SvcV5.
\end{abstract}

Keywords: architecture design, service discovery, SvcV5, operational activity dependency

\section{Introduction}

Architecture is the method, principle and guideline that guide the design of information systems. DODAF serves as a framework for guiding the defense department of the U.S.A and standardizes the specific methods and processes for designing the architecture in the military field. DODAF2.0 formed eight types of views, 52 kinds of products, from different angles to guide the contents of the architecture design, respectively [1]. Among them, SvcV5 (Operational activity to services trace-ability matrix) is the mapping matrix of service view products to operational view products. The development of this product is the key that transfer from business process to service design. However, DODAF2.0 do not give the specific design method for the product. In response to this problem, we studied how to design SvcV5 based on the existing architecture products.

SvcV5 design is about the mapping between operational activities and services, to choose which services support the execution of operational activities. In this process, service discovery and confirmation are required. Currently, in the field of service discovery methods, the research mainly focuses on service discovery based on business processes and SOA. In general, the methods for service discovery can be classified into three types: the first is a top-to-bottom method based on domain decomposition, the business elements are decomposed to a certain granularity, which is called service. The existing methods include Process-based service discovery [2], capability-based service discovery [4], Data-based service discovery [5].The second is based on the analysis of the existing system based on the method of analysis, mainly through the analysis of existing information system functions and extract some functions and as a service, these services can be reused; the third is the combination of bottom-up and up-bottom approaches, these methods take into account the need of business and the capabilities that information system functions can

${ }^{+}$Corresponding author.

snowing1124@126.com 
provide [7] . For these three service discovery methods, the first one is biased towards the business needs. While the other two approaches consider existing application sets and standards, they do not take into account the importance of business or operational activities in service discovery. We adopt the first method, drawing on the P2S (Process to Service) method [8] in the field of software engineering, study specific methods that support the design of SvcV5 products to provide decision support for architecture designers. This paper is arranged as follows: First of all, the concepts of service and combat activities are introduced. Then, based on the analysis of capability dependence and data dependency of activities, a generation method of operational activity dependency matrix is put forward. Secondly, the basic process of service discovery is proposed, including two aspects: one is the initial generation of services based on the information flow of operational activities, the other is the consolidation of service composition based on cohesion and coupling index. Finally, the SvcV5 generation process based on the common service list is proposed.

\section{SvcV5 Design Process and Relative Concepts}

Data in architecture is often related to each other. In designing the mapping of service to operational activities (SvcV5), three products are required: state transition description (OV6b), capability dependencies (CV4), and Capability to Operational activities mapping (CV6). First, the dependency analysis of operational activities was conducted. OV6b identify the relationship among operational activities based on the data. CV4 and CV6 identified the relationship among operational activity based on capability. Then, based on the information flow between operational states in OV6b, an initial service generation method based on information flow is proposed. Finally, the service cluster is updated and the mapping relation matrix of operational activity and service ( $\mathrm{SvcV5})$ is generated.

During SvcV5 design, the related concepts and their formal definitions are as follows:

\section{Define operational activity:}

$a_{i}=\left\{d_{a_{i}}, I N_{a_{i}}\right.$, Out $\left._{a_{i}}, r_{a_{i}}, C R U D_{t_{i}}\right\}$

Among them, $d_{a_{i}}$ is the description of operational activity $t_{i}$, and $I N_{a_{i}}$ presents the input of operational activities, $\mathrm{Out}_{a_{i}}$ indicating the output of operational activities. The contents of input and output is the operational information. The operational information can be described in text. CRUD is the CRUD operation of $a_{i}$, CRUD is related to $I N_{a_{i}}$ and $O u t_{a_{i}}$. There can be multiple CRUDs for a single operational activity, and activity can create $(C)$, read $(R)$, update $(U)$ and delete $(D)$ information.

In the information systems, service is achieved through a series of encapsulation of system functions, provided by service providers to service consumers through service interfaces, or available results to accomplish actual operational tasks or operational activities. In this paper, we define service:

$s_{i}=\left\{d_{s_{i}}, I_{s_{i}}\right.$, Out $\left._{s_{i}}, r_{s_{i}}, C R U D_{s_{i}}\right\}$

The service includes a description, the input operational information, the output operational information, and the CRUD operations involved. Services can be expressed as a cluster of activities. The service generated by the process of clustering activities generated. The input of the service is the union of the inputs of all the operational activities in the cluster. The output of the service is the union of the outputs of all the operational activities in the cluster. The CRUD of the service is the union of the CRUD operations of all the operational activities in the cluster.

\section{Activities Dependency Relationship Analysis}

Based on the definition of operational activities and services, we can cluster the operational activities to generate service collections. Based on the existing architecture design products, the relevant design data can be extracted (mainly from OV6b, CV4 and CV6) to establish the operational activity dependency matrix. Here consider two kinds of dependencies (based on data and capacity). The purpose of establishing the matrix of operational activities dependency matrix is to analyze the internal relations of operational activities and to provide support for establishing the objective function in the service discovery algorithm of Section 4.

There are two kinds of dependencies between operational activities $a_{i}$ and $a_{j}$ : capability dependence and data dependence. The dependencies of capability and data between operational activities $a_{i}$ and $a_{j}$ are recorded as $\Psi_{c}(i, j)$ and $\Psi_{d}(i, j)$. According to the definition of inter-activity dependencies, we can 
calculate all the dependency values. Each information object is uniquely created during operational process and then be read, updated, deleted, etc. by other activities. According to OV6b, CRUD relation matrix can be automatically generated. As shown in Table 1.

Table 1: Data analysis example of OV6b

\begin{tabular}{|c|c|c|c|c|c|}
\hline Activities & Description & Operational node & $\begin{array}{l}\text { Input } \\
\text { information }\end{array}$ & $\begin{array}{l}\text { Output } \\
\text { information }\end{array}$ & Relative CRUD \\
\hline $\begin{array}{l}a_{1}: \text { Target } \\
\text { Identificati } \\
\text { on }\end{array}$ & $\begin{array}{ll}\text { Identify } & \text { the } \\
\text { target's } & \\
\text { information } & \end{array}$ & $\begin{array}{l}\text { Radar detection } \\
\text { node, } \\
\text { Photoelectric } \\
\text { detection node }\end{array}$ & none & $\begin{array}{l}\text { target location } \\
\text { information, } \\
\text { Feature } \\
\text { Information }\end{array}$ & $\begin{array}{l}\mathrm{C} \text { (target location } \\
\text { information,Feature } \\
\text { information) }\end{array}$ \\
\hline $\begin{array}{l}a_{2}: \text { Target } \\
\text { tracking }\end{array}$ & $\begin{array}{l}\text { Target tracking } \\
\text { information }\end{array}$ & $\begin{array}{l}\text { Radar detection } \\
\text { node, } \\
\text { Photoelectric } \\
\text { detection node }\end{array}$ & $\begin{array}{l}\text { Target } \\
\text { location } \\
\text { information }\end{array}$ & $\begin{array}{l}\text { Target location } \\
\text { information, } \\
\text { Feature } \\
\text { information }\end{array}$ & $\begin{array}{l}\mathrm{R} \text { (target location } \\
\text { information,Feature } \\
\text { information) } \\
\mathrm{U} \text { (target location } \\
\text { information,Feature } \\
\text { information) }\end{array}$ \\
\hline$\ldots \ldots$. & $\ldots \ldots \ldots$ & $\ldots \ldots$ & $\ldots \ldots$ & $\ldots \ldots$ & $\ldots \ldots$ \\
\hline
\end{tabular}

Data-based dependencies can be analyzed from OV6b. We define combat activity:

$$
\Psi_{d}\left(a_{i}, a_{j}\right)=2 \frac{\left\|\operatorname{both}\left(a_{i}, a_{j}\right)\right\|}{\left\|\operatorname{Out}\left(a_{i}\right)+\operatorname{In}\left(a_{j}\right)\right\|}
$$

$\|$ both $\left(a_{i}, a_{j}\right) \|$ represents the number of data categories that are available both in $a_{i}$ and $a_{j}$. $\operatorname{Out}\left(a_{i}\right)$ Indicates the number of output data categories of $a_{i}, \operatorname{In}\left(a_{j}\right)$ indicating the number of data categories that entered $a_{j}$. This leads to a correlation matrix between activities based on the data relationship. If there is a data dependency between $a_{i}$ and $a_{j}$, corresponding to the CRUD relationship explained as: the object that created in the activity $a_{i}$ at least to be used, updated or deleted at least once in activity $a_{j}$. According to the calculation of Equation 1, the matrix of the data dependency between two activities $\Psi_{d}\left(a_{i}, a_{j}\right)$ can be calculated. In table 1 :

$$
\Psi_{d}\left(a_{1}, a_{2}\right)=2 * \frac{1}{3}=\frac{2}{3}
$$

The second matrix $\Psi_{c}\left(a_{i}, a_{j}\right)$ is based on the CV4 and CV6. CV6 reflects the mapping relationship between operational activities and capabilities, and CV4 indicates the capability correlation matrix. If the capabilities that two different activities support is correlated in CV4, Then these two activities also have dependency. The specific process is shown by the Algorithm 1. Among them, CCMatrx is capability correlation matrix according to CV4. ACMatrix is a mapping matrix of activities and capabilities, which is obtained according to CV6.

Algorithm 1: matrix generation method based on the capability dependency of the operational activities //Inputs:AC Matrix from CV4, CCMatrix from CV6, Activities set $T$

//Output: $\Psi_{c}\left(a_{i}, a_{j}\right)$

1: For all Activity $a_{i} \in T$, do

2: $\quad$ ReqSet $=\varnothing$

3: $\quad$ For all requirements pair $C_{i}, C_{j}$, do

4: $\quad$ If $[A C]_{i, x}=[A C]_{j, y}=$ relative, then

5: $\quad$ ReqSet $=$ ReqSet $\bigcup[C C]_{x, y}$

6: $\quad$ End if

7: $\quad$ End for

8: $\quad \Psi_{c}\left(a_{i}, a_{j}\right)=\operatorname{Max}(x), x \in$ ReqSet

9: End for 
The dependency relationship of operational activities $\Psi\left(a_{i}, a_{j}\right)$ can be represented as:

$$
\Psi\left(a_{i}, a_{j}\right)=\alpha \bullet \Psi_{c}\left(a_{i}, a_{j}\right)+\beta \bullet \Psi_{d}\left(a_{i}, a_{j}\right)
$$

$\alpha$ and $\beta$ are coefficients of two dependency matrices. Specific values of $\alpha$ and $\beta$ are set based on the importance of matrices and the designer's experience. In general, if the service settings are more focused on the exchange of data between operational activities, the value of $\beta$ is set to a larger one, and the setting of $\alpha$ will be larger if the focus is more on support for capabilities.

\section{Initial Service Generation Based on Information Flow}

The principle of the initial generation of service is based on the information flow through operational activities. A information flow can be created, read, updated and deleted in the operational activities processes. We assume that a data can only be created in a service, according to where the data be created (find the corresponding activities), then the activities can be clustered as a service. The process of initial determination of services is: Firstly, for each data in information flow that be created, we determine a service; then, we take the expansion of service content, find the data dependencies that is direct connected to activities, join that activity into the same service. Here, the direct relationship refers to a direct correlated line in OV6b. Finally, the remaining operational activities were identified as services.

\section{Updated Services by Cohesion and Coupling Indicators}

The initial generation of service is based on the interaction of information flow, only consider data dependency and direct correlation among services, did not take into account the service function indicators, such as cohesion and coupling. As shown in Equation 3, The cohesion of a defined service is the sum of the matrix values of the operational activities dependencies divided by the number of combinational activities. Among them, $|s|$ indicates the number of services. $C_{|S|}^{2}$ indicates the number of combinations of two services.

$$
\operatorname{coh}(S)=\left\{\begin{array}{l}
\frac{\sum \Psi\left(a_{i}, a_{j}\right)}{C_{|S|}^{2}} \forall a_{i}, a_{j} \in S ;|S|>1 \\
1 ;|S|=1
\end{array}\right.
$$

Coupling is defined as the sum of the operational activity dependencies in the service divided by the number of operational activities in the service, calculated in Equation 4.

$$
\operatorname{coup}\left(S_{1}, S_{2}\right)=\frac{\sum_{i, j}^{\prime} \Psi\left(a_{i}, a_{j}\right)}{\left|S_{1}\right| \bullet\left|S_{2}\right|} \forall a_{i} \in S_{1} \text { and } \forall a_{j} \in S_{2}
$$

According to Equation 3 and Equation 4, we can calculate the cohesion of all services and the coupling between services. In order to determine which services are selected for consolidation, an objective function $\Omega$ of consolidation is defined in Equation 5. $\Omega$ indicates both the degree of coupling and the cohesion of the service discovery. $p \operatorname{coh}(B P)$ is the cohesion of the operational activity process, defined as the cohesion of all services divided by the amount of service $|\Sigma|$ in the operational activity process. $p \operatorname{coup}(B P)$ is the degree of coupling of operational activity processes defined as the sum of service coupling divided by the number of pairs of services in the operational activity process. $\Omega$ is the ratio of cohesion and coupling, and is also the objective function of the service improvement algorithm. The larger of the objective function, the better.

$$
\begin{gathered}
\Omega=\max \frac{p \operatorname{coup}(B P)}{p \operatorname{coh}(B P)} \\
\operatorname{pcoh}(B P)=\frac{\sum \operatorname{coh}\left(S_{i}\right)}{|\Sigma|}
\end{gathered}
$$




$$
\operatorname{pcoup}(B P)=\left\{\begin{array}{l}
\frac{\sum_{i, j} \operatorname{coup}\left(S_{i}, S_{j}\right)}{\frac{|\Sigma| \bullet(|\Sigma|-1)}{2}} ;|\Sigma|>1 \\
1 ;|\Sigma|=1
\end{array}\right.
$$

\section{SvcV5 Generation}

According to the process of service generation in section 3 and section 4 , we can find out which operations are supported by the service, and then obtain the mapping between operations and services (SvcV5). In order to achieve the exact mapping relationship between the obtained operational activity and the service, we also need to find a suitable service according to the common service list and write the service name corresponding to the list into the service description. The common service list is one kind of common reference resources in DoDAF2.0. If corresponding service can not be found, the service cluster is split until the designers find corresponding service. Finally, we can write the result of the service into architecture data to form the SvcV5 product. The matrix of mappings generated by operational activities and services is shown in Table 2. $" \sqrt{ } "$ indicates the existing mapping.

Table 2: A example of SvcV5

\begin{tabular}{|l|c|c|c|c|c|c|}
\hline & $\begin{array}{c}\text { Radar } \\
\text { reconnaissance } \\
\text { services }\end{array}$ & $\begin{array}{c}\text { Photoelectric } \\
\text { surveillance } \\
\text { services }\end{array}$ & $\begin{array}{l}\text { Command } \\
\text { and decision } \\
\text { services }\end{array}$ & $\begin{array}{l}\text { Laser } \\
\text { interception } \\
\text { services }\end{array}$ & $\begin{array}{l}\text { Web } \\
\text { interception } \\
\text { services }\end{array}$ & $\begin{array}{l}\text { Radio } \\
\text { interference } \\
\text { services }\end{array}$ \\
\hline $\begin{array}{l}\text { Target } \\
\text { reconnaissance }\end{array}$ & $\sqrt{ }$ & $\sqrt{ }$ & & & & \\
\hline Target Tracking & $\sqrt{ }$ & $\sqrt{ }$ & & & & \\
\hline Information fusion & & & $\sqrt{ }$ & & \\
\hline $\begin{array}{l}\text { Command and } \\
\text { decision }\end{array}$ & & & $\sqrt{ }$ & & \\
\hline Program generation & & & & $\sqrt{ }$ & \\
\hline Laser interception & & & & & & \\
\hline $\begin{array}{l}\text { Web bomb } \\
\text { interception }\end{array}$ & & & & & & $\sqrt{ }$ \\
\hline Radio intercept & & & & & & \\
\hline
\end{tabular}

\section{Conclusion}

SvcV5 design method based on service discovery is a semi-automatic design approach. The key point is to extract the relevant information from the existing architecture design products OV6b, CV4 and CV6, then, we analyzed the relationship between operational activities and established the dependency matrix of operational activities: including data-based dependency matrix and capability-based dependency matrix. By analyzing the relationship of information flow in operational activities, an initial generation method of service based on information flow was proposed. Service cohesion and coupling index are considered and the updating method was proposed. Finally, according to the result of the clustering and the mapping of the common service list, the service in the architecture design is confirmed and the generation of SvcV5 can be done. Due to space limitations, the article did not introduce a specific case, and did not compared with other service discovery methods, much deeper study on this method will be carried out in the future.

\section{References}

[1] DoD Architecture Framework Working Group . DoD Architecture Framework Version 2.0 Volume II: Architectural Data and Models[R]. U.S.: Department of Defense, 2009.

[2] Mani, Senthil, Vibha S. Sinha, Noi Sukaviriya, and Thejaswini Ramachandra. Using user interface design to enhance service identification[C]. In ICWS' 08, IEEE International Conference on Web Services. 2008: 78-87.

[3] Levi, Keith, and Ali Arsanjani. A goal-driven approach to enterprise component identification and specification. Communications of the ACM[J] 2002,45, no. 10:45-52.

[4] Huergo, Rosane S., Paulo F. Pires, and Flavia C. Delicato. A method to identify services using master data and artifact-centric modeling approach. In Proceedings of the 29th Annual ACM Symposium on Applied 
Computing[C].2014. pp. 1225-1230.

[5] Ali Taei Zadeh, Shahnorbanun Sahran, Muriati Mukhtar, et.al. Automated Service Identification Methods: A Review[J].International Journal on Advanced Science Engineering Technology. 2016,6(6):1053-1059.

[6] Ali Taei Zadeh, Muriati Mukhtar, Shahnorbanun Sahran, et.al.Infrastuctural Phases of Automated Service Identification Framework(ASIF)[J]. Journal of Theoretical and Applied Information Technology. 2016, 83(3):451464.

[7] Devis Bianchini, Cinzia Cappiello, Valeria De Antonellis, and Barbara Pernici, Service Identification in Interorganizational Process Design[J].IEEE TRANSACTIONS ON SERVICES COMPUTING, VOL. 7, NO. 2,2014.pp: 265-278. 